The Effect of Excess Carbon on the Crystallographic, Microstructural, and Mechanical Properties of CVD Silicon Carbide Fibers

J. V. Marzik, W. J. Croft, R. J. Staples, W. J. MoberlyChan

December 11, 2006

Materials Research Society

Boston, MA, United States

November 26, 2006 through December 1, 2006 
This document was prepared as an account of work sponsored by an agency of the United States Government. Neither the United States Government nor the University of California nor any of their employees, makes any warranty, express or implied, or assumes any legal liability or responsibility for the accuracy, completeness, or usefulness of any information, apparatus, product, or process disclosed, or represents that its use would not infringe privately owned rights. Reference herein to any specific commercial product, process, or service by trade name, trademark, manufacturer, or otherwise, does not necessarily constitute or imply its endorsement, recommendation, or favoring by the United States Government or the University of California. The views and opinions of authors expressed herein do not necessarily state or reflect those of the United States Government or the University of California, and shall not be used for advertising or product endorsement purposes. 


\title{
The Effect of Excess Carbon on the Crystallographic, Microstructural, and Mechanical Properties of CVD Silicon Carbide Fibers
}

\author{
James V. Marzik ${ }^{1}$, William J. Croft ${ }^{2}$, Richard J. Staples ${ }^{2}$, and Warren J. MoberlyChan ${ }^{3}$ \\ ${ }^{1}$ Specialty Materials, Inc., Lowell, MA \\ ${ }^{2}$ Harvard University, Cambridge MA \\ ${ }^{3}$ Lawrence Livermore National Laboratory, Livermore, CA
}

\begin{abstract}
Silicon carbide (SiC) fibers made by chemical vapor deposition (CVD) are of interest for organic, ceramic, and metal matrix composite materials due their high strength, high elastic modulus, and retention of mechanical properties at elevated processing and operating temperatures. The properties of SCS- ${ }^{\mathrm{TM}}$ silicon carbide fibers, which are made by a commercial process and consist largely of stoichiometric $\mathrm{SiC}$, were compared with an experimental carbonrich $\mathrm{CVD} \mathrm{SiC}$ fiber, to which excess carbon was added during the CVD process. The concentration, homogeneity, and distribution of carbon were measured using energy dispersive $\mathrm{x}$-ray spectroscopy (SEM/EDS). The effect of excess carbon on the tensile strength, elastic modulus, and the crystallographic and microstructural properties of CVD silicon carbide fibers was investigated using tensile testing, x-ray diffraction, scanning electron microscopy (SEM), and transmission electron microscopy (TEM).
\end{abstract}

\section{INTRODUCTION}

Chemically vapor deposited (CVD) silicon carbide fibers [1] have been commercially produced for over two decades as a composite reinforcement for a number of structural aerospace applications. The high-temperature stability of silicon carbide makes it particularly attractive as the reinforcement for metal and ceramic matrix composites. SCS-6 ${ }^{\mathrm{TM}}$ silicon carbide has been investigated as the load-bearing reinforcement in composites including SiC/titanium, $\mathrm{SiC} /$ aluminum, and $\mathrm{SiC} / \mathrm{Si}_{3} \mathrm{~N}_{4}$. The CVD process is particularly amenable to tailoring the composition in the fiber. Thus, the $\mathrm{Si} / \mathrm{C}$ compositional ratio can be varied in different regions of the deposition. Fibers (designated as SCS-6 and C-rich) with two significantly different Si/C ratios were investigated in this work.

\section{EXPERIMENTAL}

\section{$\underline{\text { SiC fiber synthesis }}$}

The silicon carbide fibers were synthesized using a commercial CVD process [1] for SCS-6 and a modification thereof for the C-rich SiC. A $33 \mu$ m diameter carbon monofilament was drawn continuously through a 2 -meter vertical glass reactor that was sealed at the top and bottom by pools of mercury. The mercury formed both an electrical contact and a gas seal. Initially, a thin $(\sim 1 \mu \mathrm{m})$ coating of pyrolytic graphite was deposited onto the carbon substrate. A mixture of alkylchlorosilanes, hydrogen, and propane was fed into the reactor, partially consumed during the deposition process, and exhausted along with byproduct gases to a gas separation and recycling facility. The filament was resistively heated to $1300-1400^{\circ} \mathrm{C}$ by application of a DC voltage to the mercury seals. The deposition material was primarily $\beta$-SiC, the cubic polymorph. In SCS-6, the $\mathrm{SiC}$ exhibited a columnar structure with a growth pattern 


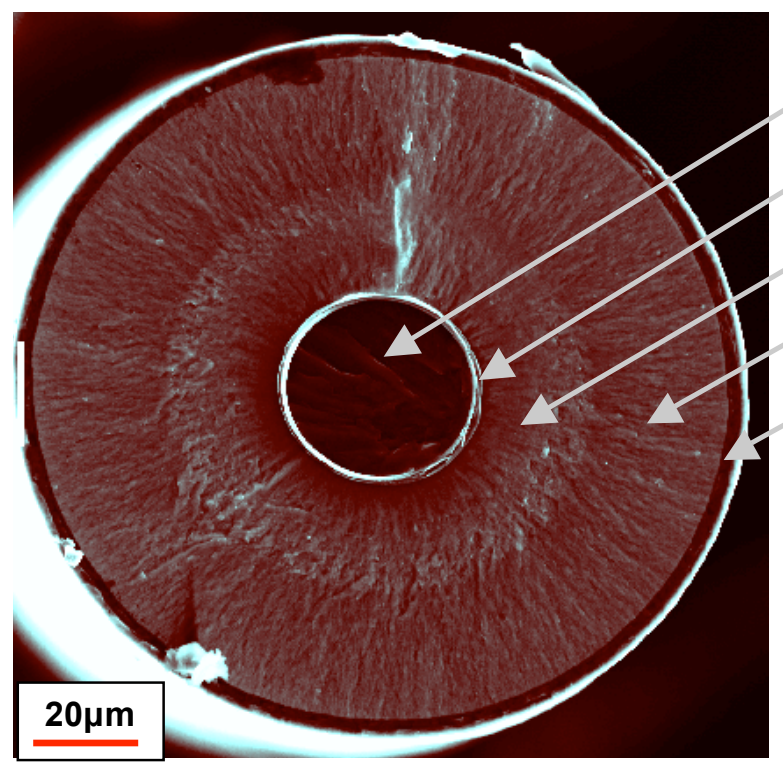

\section{Carbon monofilament substrate}

Pyrolytic graphite

$1^{\text {st }} \mathrm{SiC}$ deposition zone

$2^{\text {nd }} \mathrm{SiC}$ deposition zone

Carbon-rich coating

Figure 1. SEM micrograph of cross-sectional fracture surface of the SCS-6 ${ }^{\mathrm{TM}} \mathrm{SiC}$ fiber showing the carbon substrate and various deposition zones.

extending radially from the substrate. Approximately midway through deposition process, the growth of $\mathrm{SiC}$ was re-nucleated. This results in the observation of a mid-radius boundary when the fiber is examined in cross section (Figs. $1 \& 2$ ). A $1-2 \mu$ m carbon-rich coating was deposited in the final region of the reactor tube. The coating was compositionally graded, starting as nearly pure carbon and increasing in silicon content towards the outer surface. Two such coatings were deposited onto the SCS- 6 fiber. The diameter of the silicon carbide was $\sim 140 \mu \mathrm{m}$ and the draw rate of the filament through the reactor was $3-5 \mathrm{~m} / \mathrm{min}$ with the $\mathrm{SiC}$ product being spooled continuously at the reactor exit point. $\mathrm{C}$-rich $\mathrm{SiC}$ fibers were prepared similarly to SCS-6 fibers described above, with the addition of excess hydrocarbons to the reaction mixture.

\section{Characterization and testing techniques}

SEM/EDS analyses were carried out on a digital SEM (JEOL 5610), and a ThermoNoran System Six EDS system. TEM sample preparation was performed using an FEI NOVA600 DualBeam FIB/SEM (focused ion beam). TEM analyses were performed on an FEI CM300-FEG (field emission gun) microscope operating at 300keV and using a Gatan energy filter/CCD imaging system. Electron energy loss spectroscopy (EELS) analysis was performed using the Gatan GIF, and EDS analysis was performed in scanning transmission (STEM) mode using an Oxford detector and Emispec ESVision software. X-ray diffraction (XRD) patterns were obtained using a Scintag Model XDS-2000 x-ray diffractometer calibrated with a NIST silicon standard. Diffraction patterns were taken using copper K $\alpha$ radiation on samples consisting of six parallel 1-inch fiber segments arrayed on a quartz plate. Data taken with the fibers both parallel and perpendicular to the beam showed no observed difference between the two sample positions. Individual fibers were also examined using a Bruker 3-circle x-ray goniometer with the x-ray beam (Mo radiation) perpendicular to the fiber axis. The fiber was stationary during the exposure and the data was collected using an area detector. The SiC fibers were tested for tensile strength and modulus on an Instron universal testing machine. Ultimate tensile strengths were measured using ten tests at a 1-inch gauge length. Tensile modulus was measured using three tests at a 12inch gauge length. Modulus was calculated using a curve fit in the stress-strain curve from 200$800 \mathrm{MPa}$. 

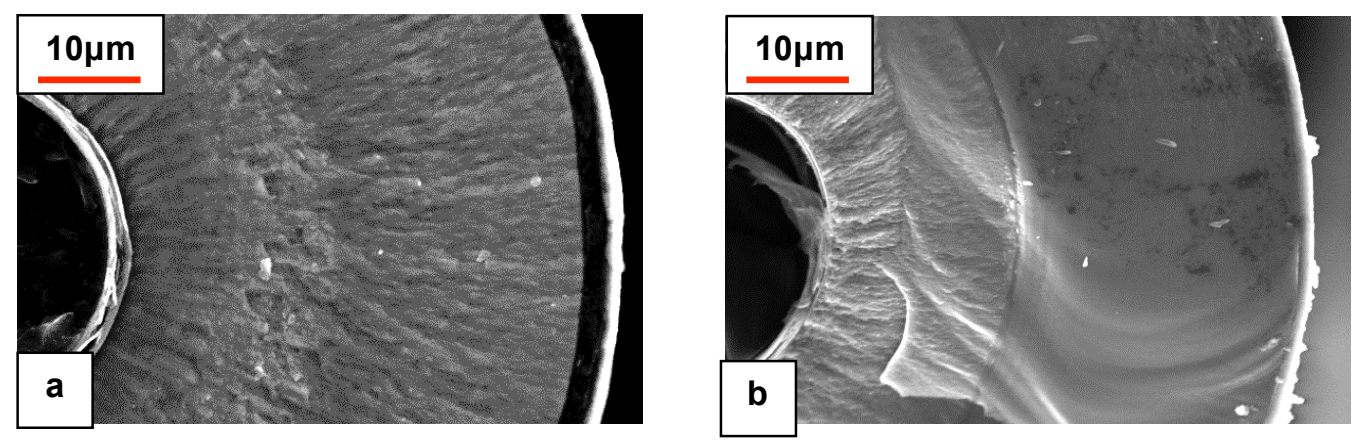

Figure 2. SEM micrographs showing fracture surfaces of SCS-6 ${ }^{\mathrm{TM}} \mathrm{SiC}$ (a) and carbonrich $\mathrm{SiC}(\mathrm{b})$ fibers. The second SiC deposition of the SCS-6 fiber shows columnar grain growth radiating from the substrate to the surface whereas the grain structure of the $\mathrm{C}$ rich $2^{\text {nd }}$ deposition zone of $\mathrm{SiC}$ growth is relatively featureless.

Table 1. Mechanical properties of SiC fibers

$\begin{array}{cccc}\text { Fiber type } & \text { Sample \# } & \text { Tensile strength } \mathbf{( k P a )} & \text { Tensile Elastic modulus (GPa) } \\ \text { SCS-6 } & 1 & 3990 & 402 \\ \text { SCS-6 } & 2 & 3920 & 352 \\ \text { C-rich } & 3 & 1230 & 232 \\ \text { C-rich } & 4 & 670 & 217\end{array}$

\section{RESULTS AND DISCUSSION}

Figure 1 shows a cross-sectional fracture surface of an SCS-6 ${ }^{\mathrm{TM}}$ fiber. The deposition zones described in the experimental section are labeled. Micrographs in Fig. 2 compare the grain structure of SCS-6 and C-rich SiC. The second SiC deposition of the SCS-6 fiber shows columnar grain growth radiating from the substrate to the surface whereas the grain structure of the $\mathrm{C}$-rich $2^{\text {nd }}$ deposition zone of $\mathrm{SiC}$ growth is relatively featureless. EDS analysis of this $2^{\text {nd }}$ deposition zone showed a composition close to stoichiometric SiC in SCS-6, which is consistent with a previous report [2]. The $\mathrm{C}$-rich $\mathrm{SiC}$ fibers had a significantly higher carbon concentration (20-30 relative atomic percent higher in carbon). The addition of excess carbon also had a significant effect on the mechanical properties of the fiber. The tensile strength of the C-rich fiber dropped by approximately a factor of 4-5 relative to the SCS-6 fiber and the tensile elastic modulus of the C-rich fiber dropped roughly by a factor of 2 relative to SCS- 6 . The tensile mechanical properties are shown in Table 1. Figure 3 shows XRD patterns of SCS-6 (3a) and Crich $\mathrm{SiC}$ fibers $(3 \mathrm{~b})$ taken on a powder diffractometer. The sharper diffraction peaks observed in samples of SCS-6, coupled with a higher magnitude of low-angle scattering observed in samples of the C-rich $\mathrm{SiC}$, are consistent with a higher degree of crystallinity in SCS-6 relative to the Crich SiC fibers. The 111, 200, and 220 reflections were observed in SCS-6. An additional peak was assigned to graphite 002 from the carbon substrate. XRD diffraction rings with brighter and darker segments in the SCS-6 fiber are indicative of grain orientation in the direction perpendicular to the fiber axis. The C-rich fiber (inset, Fig. 3b) showed more diffuse rings with 


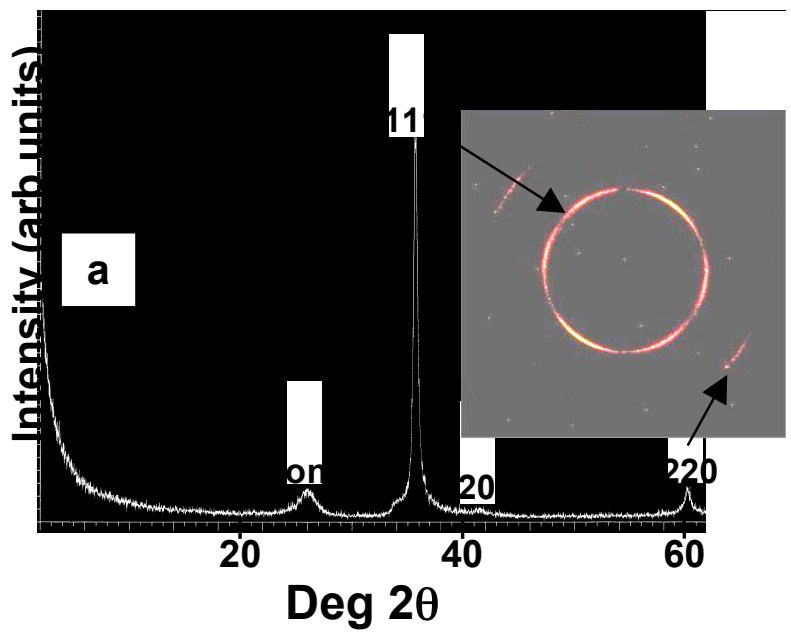

\section{Carbon-rich SiC}

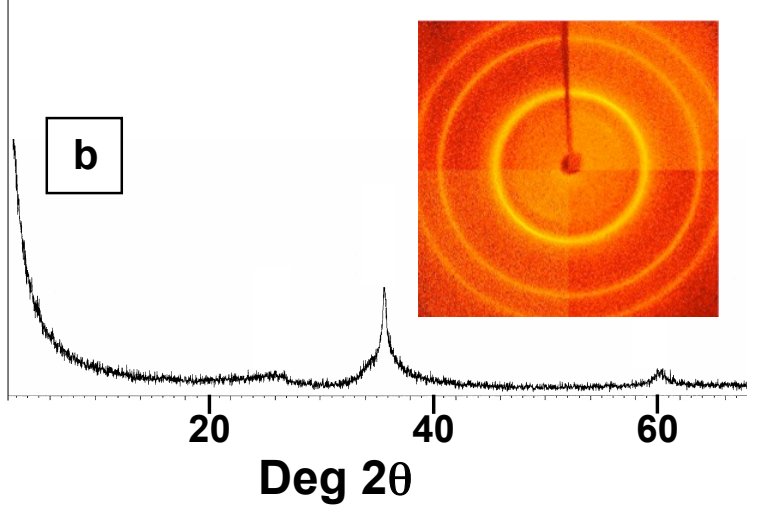

Figure 3. X-ray diffraction patterns of SCS-6 ${ }^{\mathrm{TM}}$ (a) and carbon-rich $\mathrm{SiC}$ fibers (b). The sharper diffraction peaks observed in samples of SCS-6, coupled with a higher magnitude of low-angle scattering observed in samples of the carbon-rich $\mathrm{SiC}$, are consistent with a higher degree of crystallinity in SCS-6 relative to the C-rich SiC fibers. The insets show XRD patterns taken on a 3-circle goniometer with corresponding reflections indicated by arrows.

no evidence of orientation. The higher background intensity is attributed to more scatter, consistent with a lower degree of crystallinity in the C-rich $\mathrm{SiC}$ fiber.

Figures $4 \mathrm{a}$ and $4 \mathrm{~b}$ are SEM images acquired in the DualBeam FIB/SEM during TEM sample preparation. The lower magnification image depicts the $\mathrm{SiC}$ fiber attached to a TEM grid. Also it images the gas injection system (GIS) needle inserted to apply a protective Pt-CVD coating prior to FIB cross-sectioning. Often FIB sample preparation results in artifacts parallel to the electron beam, commonly referred to as "curtaining". The directionality of such artifacts often limits the ability to see microstructural features with a similar orientation in the TEM images. Thus the fiber/TEM sample was deliberately oriented with a $30^{\circ}$ inclination to the FIB. As shown in the higher magnification image, this leads to FIB trenches that are at an angle. However, the FIB polished surfaces still exhibit the coating layers distinctly (in this case a double coating), as well as the underlying SiC substrate (the brighter layer at the top is the protective Pt layer which provides improved edge retention in the TEM cross sectioning process). As will be shown in the TEM images, the sample preparation was sufficient to make any curtaining effects negligible. High resolution SEM imaging of the two surfaces exhibited some differences even though both fiber types had the same coating. Both surfaces exhibited very fine $(<100 \mathrm{~nm})$ nodular features. At slightly lower magnification, gentle humps on the order of $1 \mu \mathrm{m}$ were visible on both fiber surfaces. The surface roughness of the two fiber types appeared to be conformal to the underlying SiC roughness. The SCS-6 had a significantly rougher surface than did the $\mathrm{C}$-rich $\mathrm{SiC}$.

TEM imaging and electron diffraction were performed of the FIB-prepared crosssections. No directional effects related to "curtaining" were observed in the TEM images. There were, however, fine artifacts from Ga contamination on the surfaces caused by the FIB-Ga ${ }^{+}$ion beam. FIB, as does most ion beam preparation, typically causes the surfaces of the thin TEM lamella to become amorphous. This adds some noise to the TEM images, but crystalline features are still observable. 


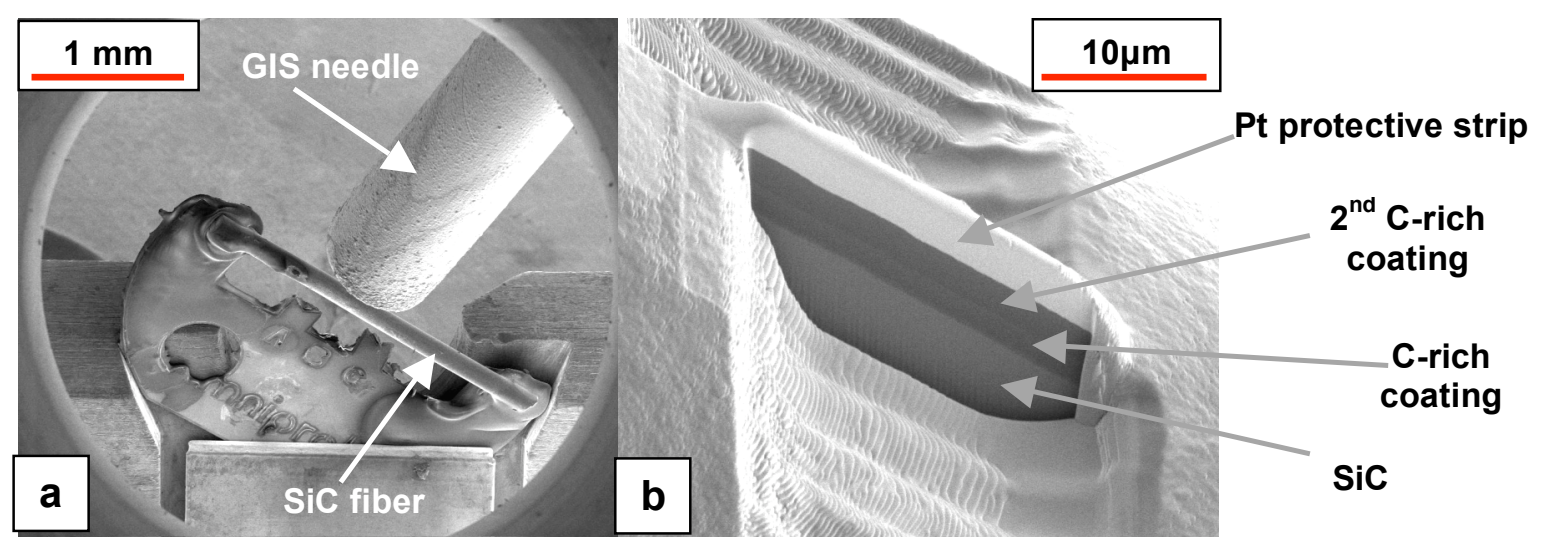

Figure 4. Focused ion beam experimental setup is shown in $4 \mathrm{a}$ (see text). $4 \mathrm{~b}$ is a micrograph of a focused ion beam trench that was milled into the surface of an SCS-6 SiC fiber.

Figure 5a is a dark field image of the SCS-6 cross section which shows the SiC to have columnar grains of 50-100nm in diameter and their heights of several microns. The dark field image was acquired using the g-vector associated with the 111-SiC normal to the substratecoating interface. Thus the grains with their 111 planes parallel to the substrate are highlighted. In addition many faults were observed. High resolution lattice imaging, not shown, indicated frequent stacking faults in the $\beta$-cubic $\mathrm{SiC}$ grains, but no evidence of hexagonal polytypes were observed. Regions of perfect $\beta$-stacking more than 5-10 planes of atoms in row were rarely observed. A double coating had been applied to this sample. Each coating was a graded layer, being mostly carbon-rich adjacent to the $\mathrm{SiC}$ with an increase in Si concentration nearer the outer fiber surface. No sign of crystallinity was observed (although the TEM may not resolve crystals smaller than 1-2nm, as the FIB samples tend to be many 10's of nanometers thick). A difference was observed, however, between the inner and outer portions of each coating layer. Dark field tends to make denser structures appear bright and less dense material appear dark. By using a combination of dark field images acquired at different g-vector orientations (not included), the inner part of the coating appeared to be less dense, consistent with it being more carbon-rich and with the outer part of the coating being more Si rich. This is consistent with previous work using EELS analysis [2]. Figure $5 \mathrm{~b}$ is a selected area diffraction (SAD) pattern acquired on a $\sim 1 \mu \mathrm{m}$ area of the $\mathrm{SiC}$. Although rings were discontinuous, the spacing consistently matched the $\beta$-SiC polycrystalline ring pattern. The intensity of the 111 ring was mostly concentrated in a short arc indicating 111-oriented crystalline texture in the $\mathrm{SiC}$, consistent with previous work [3]. Such texture effects are consistent with the XRD data in Fig 3. Fig. 5c is a bright field image of the Crich $\mathrm{SiC}$ fiber cross-section at the fiber-coating interface in which the $\mathrm{SiC}$ region exhibits no diffraction contrast. However, the rings in the SAD diffraction pattern of a $\sim 2 \mu \mathrm{m}$ area (Fig. $5 \mathrm{~d}$ ) indicated crystalline $\beta$-SiC is present. The absence of discernible grains in this FIB-prepared sample indicates that they are $<2 \mathrm{~nm}$. The extra arc rings in Fig. $5 \mathrm{~d}$ are a result of the Ga ion FIB preparation.

\section{CONCLUSIONS}

The addition of excess carbon to SiC fibers significantly affected the fiber's crystallinity, microstructure, and mechanical properties. SEM analysis of fracture surfaces indicated that the $2^{\text {nd }}$ deposition zone of the SCS-6 SiC fiber had a columnar grain growth pattern extending 


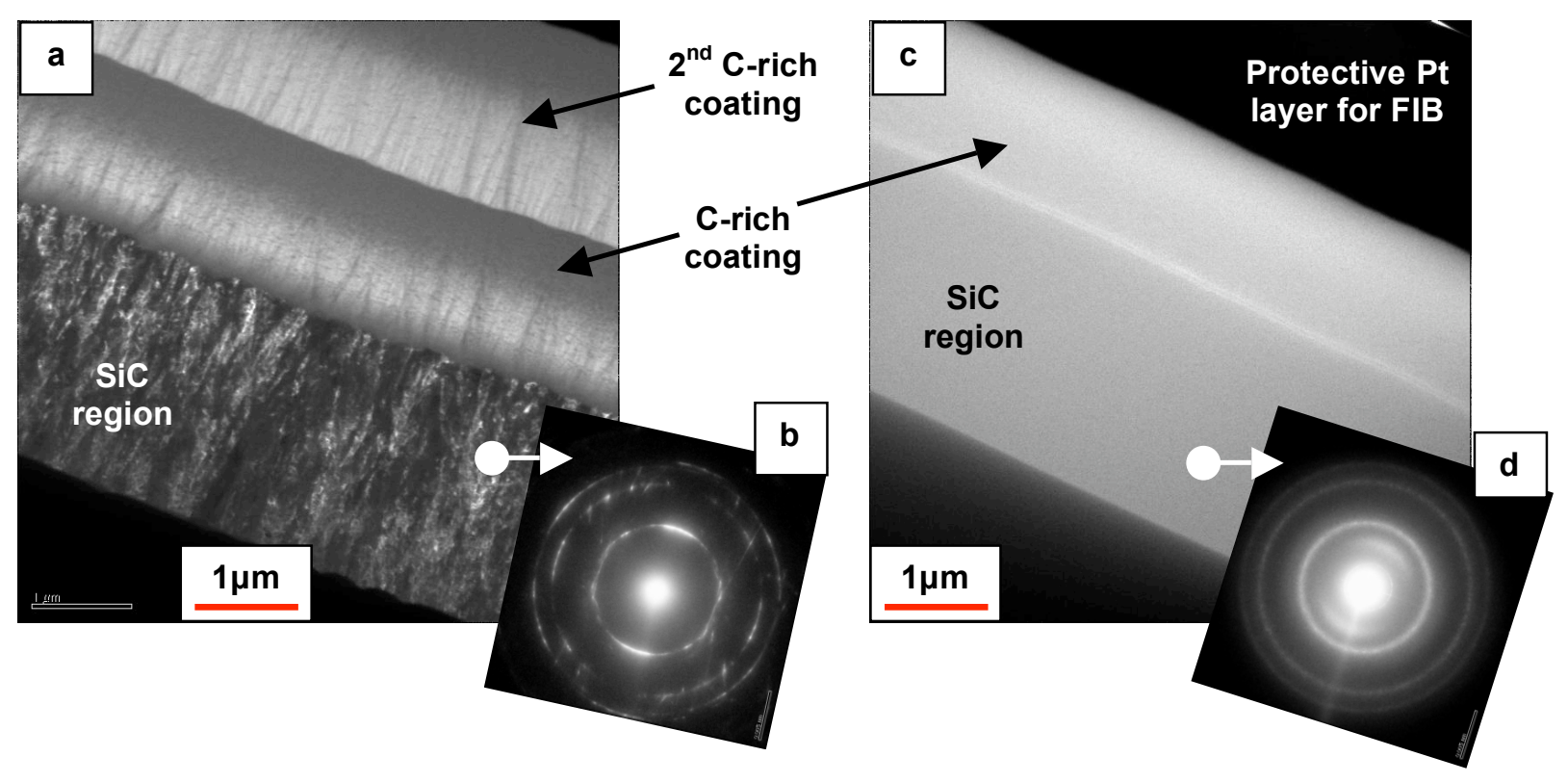

Figure 5. 5a shows a dark-field TEM of SCS-6 SiC fiber at the SiC-coating interface acquired using the g-vector associated with the 111-SiC normal to the substrate/coating interface. The upper part shows two carbon-rich coatings. 5b shows a selected area diffraction (SAD) pattern of the $\mathrm{SiC}$ region in which the rings are discontinuous and indicate a 111-texture normal to the substrate. 5c shows a bright-field TEM of the C-rich SiC fiber at the SiC-coating interface. 5d shows the $\mathrm{SAD}$ of the $\mathrm{SiC}$ region in which the rings appear mostly uniform, indicating the polycrystalline $\mathrm{SiC}$ region has no strong preferred texture.

perpendicular to and radially out from the carbon substrate. The $2^{\text {nd }}$ deposition zone of the Crich $\mathrm{SiC}$ fiber was comparatively featureless. XRD and TEM/SAD analysis indicated while both fibers consisted primarily of cubic $\beta$-SiC, the SCS- 6 SiC fibers possessed a higher degree of crystallinity relative to the C-rich $\mathrm{SiC}$ fibers. The SCS-6 SiC fibers also showed a 111-oriented texture perpendicular to the fiber axis, whereas the $\mathrm{C}$-rich $\mathrm{SiC}$ fibers possessed no discernible texture. The crystallinity and texture have an apparent effect on the mechanical properties of the fiber. The tensile strength and modulus of the SCS-6 SiC fiber was significantly higher than those of the C-rich $\mathrm{SiC}$ fiber.

\section{ACKNOWLEDGEMENTS}

This work was performed under the auspices of the United States Dept. of Energy by the University of California, Lawrence Livermore National Laboratories under contract of No. W7405-Eng-48. UCRL-PROC-226742.

\section{REFERENCES}

1. R.J. Suplinskas and J.V. Marzik, in Handbook of Reinforcements for Plastics, edited by J.V. Milewski and H.S. Katz (New York: Van Nostrand Reinhold Co., 1987) pp. 340-363.

2. X.J. Ning and P. Pirouz, J. Amer. Ceram. Soc. 76, 2033 (1993).

3. X.J. Ning and P. Pirouz, J. Mater. Res. 6, 2234 (1991). 